EPJ Web of Conferences 95,02004 (2015)

DOI: $10.1051 /$ epjconf/ 20159502004

(C) Owned by the authors, published by EDP Sciences, 2015

\title{
Direct dark matter searches review
}

\author{
Jules Gascon ${ }^{1, a}$ \\ ${ }^{1} I P N L$, Université de Lyon (Université Lyon 1) and CNRS/IN2P3
}

\begin{abstract}
Direct Dark Matter Searches are experiments looking for the energetic recoils due to the scattering of Weakly Interacting Massive Particles (WIMPs) from our galactic halo on nuclei in a terrestrial target. The principles of these type of searches is described, and the status and results of the leading experiments in that field are presented, as well as their prospects in the coming years.
\end{abstract}

\section{Introduction}

Direct searches for Dark Matter in the form of Weakly Interacting Massive Particles (WIMPs) are experiments looking for energetic recoiling ions produced by the scattering of such a particle on laboratory targets. The growing interest for these experiments stems from the continuous flow of cosmological and astrophysical observations that underlines the need for a large fraction of the mass of the Universe to come from a yet undetected form of matter that does not absorb or emit electromagnetic radiation, and has decoupled from ordinary matter and become non-relativistic early enough in the history of the Big Bang to explain the formation of large structures (for more details, see e.g. Refs. [1]). An elegant solution is provided by particles having weak-force interactions such as the neutralino acting as the lightest Supersymmetric (SUSY) particle, as it is often the case in minimal version of this model (MSSM). In this scenario, a conclusive identification could be performed by combining the results of tests of Supersymmetry at LHC with those of direct searches and other experiments looking for WIMP annihilation products in cosmic ray (indirect searches). Paradoxically, the absence of signal for the Minimal SuperSymmetric Model (MSSM) at the LHC has not reduced the domain of the direct searches. Instead, it suggest that if there are Supersymmetric particles, their mass spectrum is less constrained than in the MSSM, and opens the challenge for direct searches to design experiments that are sensitive to smaller mass or cross-section values than those predicted by this specific version of theses models. Direct searches are essential at proving that eventual WIMPs particles discovered at colliders are indeed present in the halo of our Galaxy.

The present review describes the principles of direct searches for Dark Matter, and presents the status and prospects of some of the leading currently-running experiments.

\section{Direct Searches}

Direct searches aim at measuring the rate and the energy spectrum of nuclear recoils induced by collisions with WIMPs. The four factors entering the expression of this rate are: the local WIMP density,

\footnotetext{
ae-mail: j.gascon@ipnl.in2p3.fr
} 
their velocity distribution relative to Earth, their scattering cross-section on a nucleon, and a coherence factor that takes into account that the nuclear target is made of $A$ nucleons. The uncertainties associated to these factors can be large and must be kept in mind when comparing experimental results with theoretical predictions. In order to have a consistent comparison between the sensitivity of different experiments, one generally use the conventions laid out by Lewin and Smith [2] concerning the local WIMP density and the choice of a Maxwellian velocity distribution, to which the Sun and Earth velocities are added. More complex halo models have been considered [3], and more precise evaluation of the local WIMP density are available [4], but the conventional values are still relevant for the sake of comparing experiments. However two cases can be distinguished for its scaling to a WIMP-nucleus cross-section $\sigma_{A}$. In the case of spin-dependent interactions, the scaling factor is $J(J+1)$, where $J$ is the nuclear spin. For spin-independent interactions, the factor is $A^{2}$, where $A$ is the atomic mass of the target nucleus. A kinematic factor $\sim A^{2}$ also intervenes in the definition of the cross-section. The additional contribution from nuclear form factors dampens somewhat the $A$ dependence, but in most models the spin-independent amplitude dominates, favouring target nuclei with large $A$ values. Fig. 1 compares the expected number of counts above threshold observed after a one-year exposure of $1 \mathrm{~kg}$ of different target materials, for a spin-independent cross-section of $10^{-8} \mathrm{pb}$ and a WIMP mass of 100 $\mathrm{GeV} \cdot \mathrm{c}^{-2}$, calculated using the prescriptions of Lewin and Smith [2]. Larger $A$ values are generally associated with larger rates, but the experimental threshold in recoil energy also matters. In order to be relevant for a wide range of models, searches results are expressed as excluded or preferred regions in a graph of $\sigma_{n}$ versus $M_{W}$ (Right pannel of Fig. 1). This figure displays the so-called neutrino floor [5], where the rate of nuclear recoil induced by the coherent scattering on nuclei of neutrino from the sun and cosmic rays cannot be distinguished from an eventual WIMP signal. The cross-sections generally decrease with mass.

The simplest versions of Supersymmetric models that are not excluded at the LHC tend to predict masses in the range from $100 \mathrm{GeV} / \mathrm{c}^{2}$ to few $\mathrm{TeV} / \mathrm{c}^{2}$, and cross-section values between $10^{-12}$ and $10^{-8} \mathrm{pb}[6]$. To give an idea of the corresponding rates of nuclear recoils in a detector, a crosssection of $10^{-8} \mathrm{pb}$ would yield approximately one such recoil per year per kilogram. For $10^{-11} \mathrm{pb}$, the corresponding rate would be one recoil per year per ton. This set the scale for future experiments.

In alternative models, the WIMP can have a mass between 1 and $10 \mathrm{GeV} / \mathrm{c}^{2}$. Present experiments are not putting strong constraints in this mass range. The challenge there lies in designing a detector that can efficiently detect very low energy nuclear recoils.

The main challenge in direct searches is the low expected rates, requiring an extreme suppression of natural radioactivity in the relevant recoil energy range. As a comparison, the natural radioactivity of the human body is ten orders of magnitude above the rate associated to a cross-section of $10^{-8} \mathrm{pb}$. Thus, the detector and its surroundings must be made of rigorously selected materials, with massive shielding and a protection from cosmic activation that can only be achieved in deep underground locations. What remains after this large suppression often comes from poorly understood tails of distributions and tiny detector imperfections. These are difficult to model, as present-day detectors often probe unchartered levels of low backgrounds in this domain of recoil energy. There is a strong need for techniques to further discriminate the signal from the background. The measurement of the directions of the individual nuclear recoils would open the interesting possibility of correlating them with the velocity vector of the sun relative to the Galaxy. However, the length of typical recoil tracks is $20 \mathrm{~nm}$ in solids and $30 \mu \mathrm{m}$ in low-pressure gas, and no detector has -yet- achieved the required spatial resolution with a sufficient mass and radiopurity [7]. A more subtle effect is provided by the contribution of the Earth orbital motion around the Sun to its radial velocity relative to the galactic center, resulting in a annual modulation of the WIMP flux of the order of a few \% [2]. Exploiting this signature requires a very large detector mass and long exposure [8]. In the absence of a measurement 

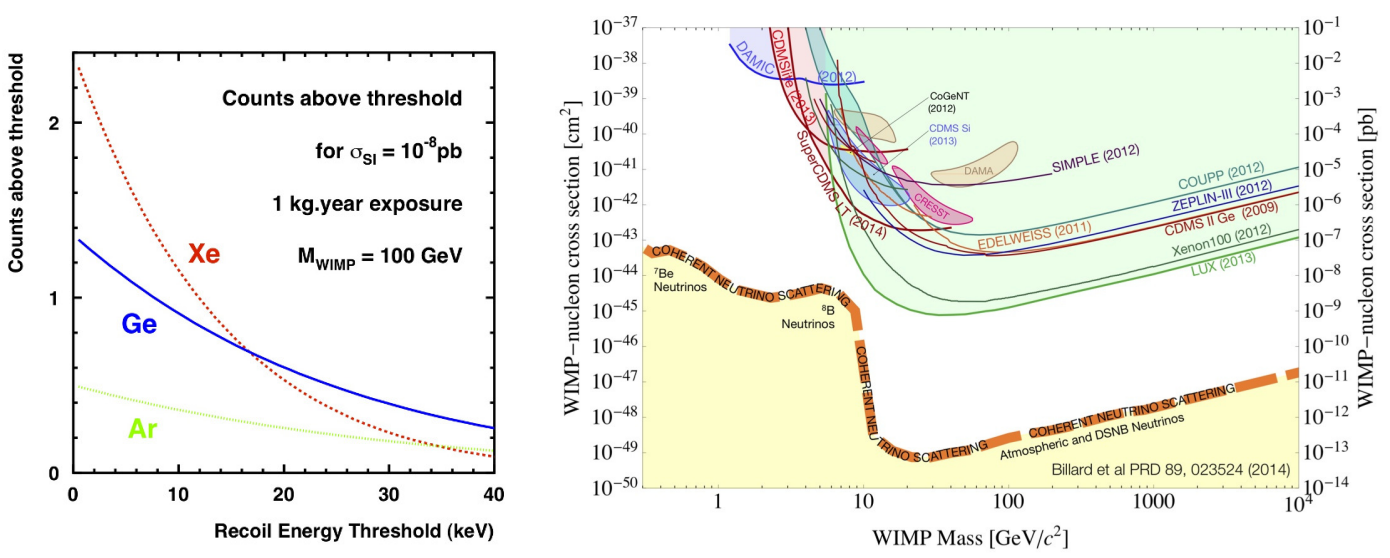

Figure 1. Left pannel: Expected number of counts above threshold as a function of this threshold, in a one-year exposure of $1 \mathrm{~kg}$ of different target materials for a spin-independent cross-section of $10^{-8} \mathrm{pb}$ and a WIMP mass of $100 \mathrm{GeV} \cdot \mathrm{c}^{-2}$, calculated using the prescriptions of Ref. [2]. Right: Exclusions limits for the spin-independent WIMP scattering cross-section as a function of the mass of the WIMP from different direct search experiments (see text). The hashed lines corresponds to neutrino floor (see text). The right pannel is taken from Ref. [5] .

of the constant part of the scattering rate, the interpretation of the modulated part depends on the details of the velocity distribution.

An important signature to establish its coherent aspect of the scattering and help establish the spin dependent or independent nature is the measurement of its dependence with $A$. This pleads for the simultaneous development of detectors with differing target nuclei. The comparison of different detectors with different systematics is also essential to confirm any discovery.

Many experiments significantly reduce the dominant background at low energy due to $\mathrm{X}$ and $\gamma$ rays and to $\beta$ decays by techniques differentiating the electron recoils produced by these processes from the sought-for nuclear recoils. It then remains to shield the experiment from the fast neutrons able to produce nuclear recoils with energies in the range of interest. In detectors with dimension larger than the $\sim \mathrm{cm}$ mean-free path of fast neutrons, this can be achieved by defining an internal fiducial volume . Alternatively, in large arrays of smaller detectors, neutron interactions can be tagged by coincidences. The discrimination between electron and ion recoils can be done by exploiting the different behavior of these particles as they slow down and stopped in the medium. The recoil energy is first converted in phonons and ionization, eventually producing scintillation light. In the end, all the energy that does not escape the detector or is not trapped in permanent defects is thermalized into low-energy phonons, producing a temperature increase. The relative yield of the three processes (phonon, ionization and scintillation) often differ for electron and nuclear recoils, and the simultaneous measurement of two of these signals can thus tag the nature of the recoil. A measurement of thermal phonons is truly calorimetric, and has a very small dependence on the recoiling particle [9]. It provides the most precise measurement of the recoil energy. Ionization and scintillation yields depend strongly on the nature or particle and can provide the needed discrimination. However the ratio of yields of nuclear and electron recoils (the so-called quenching factor) needs to be calibrated down to the required energies.

The combination of signals used by experiments having produced competitive WIMP limits in the last five years are phonon and ionization (CDMS [10], EDELWEISS [11]), heat and scintillation (CRESST [12]) and ionization and scintillation (LUX [13], XENON [14], ZEPLIN [15]). 

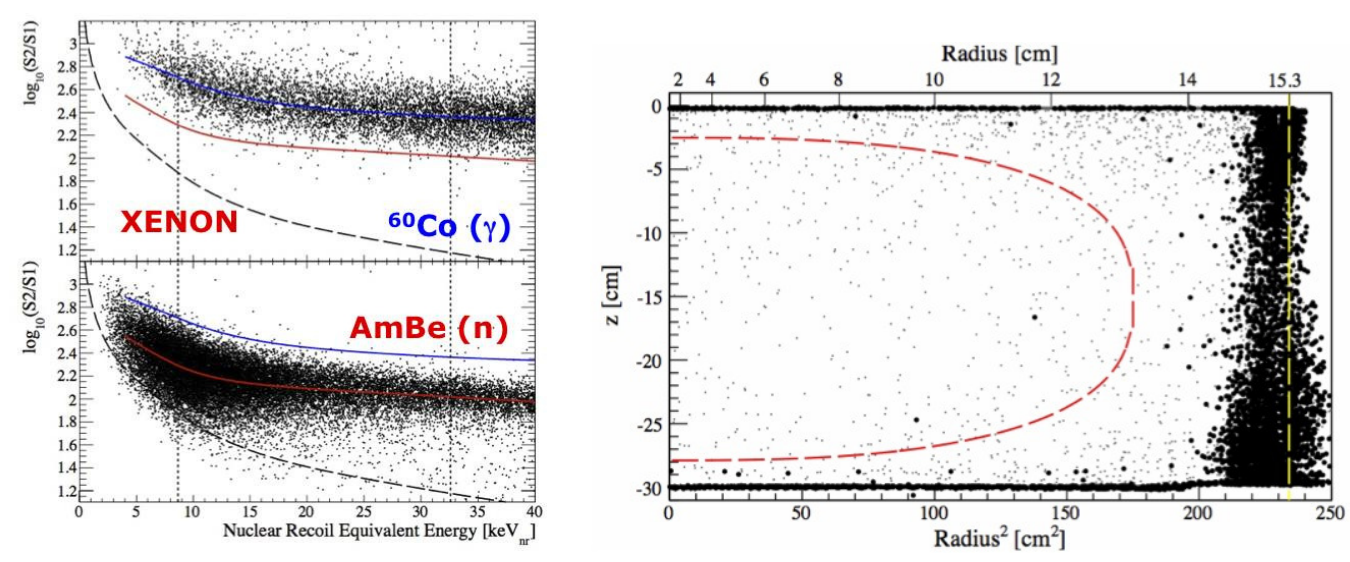

Figure 2. Left: Discrimination parameter versus recoil energy in XENON-100 (see text). The blue and red lines depicts the average ratio of the secondary signal to the primary signal (S2/S1) for electron and ion recoils, respectively. Right: corresponding radial and vertical coordinates of events in the same experiment. Both figures are taken from Ref. [14].

Alternative electron/ion discrimination techniques involve pulse shape of scintillation signals, applicable for example in liquid argon and neon [16], and in CsI [17]. The experiments COUPP, PICASSO and SIMPLE [19], for their part, use the large linear energy loss of recoiling ions to trigger bubble formation in superheated liquids. The experiment COUPP has assembled the largest such detector, with a relatively small target mass of $4 \mathrm{~kg}$ of $\mathrm{CF}_{3} \mathrm{I}$, not competitive with the denser and heavier germanium and xenon arrays, but adequate for testing models where WIMPs have spin-dependent interactions only. To probe the very low WIMP mass region, the experiment DAMIC [18] uses chargecoupled device (CCD) to identify very low-energy deposit due to nuclear recoils, and has shown test results with a few $\sim \mathrm{g}$ detectors.

In the following, we present the most recent results from the experiments LUX, XENON, CDMS, EDELWEISS and CRESST.

\section{Noble liquid searches}

The experiments LUX [13] and XENON100 [14] uses the relative ionization and scintillation yields to discriminate electron and ion recoils in, respectively, two-phase volumes of 370 and $170 \mathrm{~kg}$ of xenon. The target in the liquid phase is observed by an array of more than a hundred photomultipliers (PMs), respectively at the top and bottom of the cylindrical container. These PMs detect the primary scintillation light (signal S1) due to the de-excitation of a meta-stable excimer efficiently produced in atomic collisions. Then, an intense electric field drifts the electrons produced in ionization processes toward the top surface where they are further accelerated in the gaseous phase, creating a secondary pulse of scintillation (S2) proportional to the ionization yield.

The average ratio (S2/S1) for electron and ion recoils differ by a factor $\sim 0.4$ over most of the relevant energy range (Fig. 2). The discrimination performances are limited by the statistics of scintillation photons (a $10 \mathrm{keV}$ nuclear recoil correspond to a $\mathrm{S} 1$ of approximately 5 and 7 photoelectrons in XENON100 and LUX, respectively). The radial and vertical axis of the primary interaction are derived from the signal intensity in the different PMs and the time delay between the two pulses, re- 

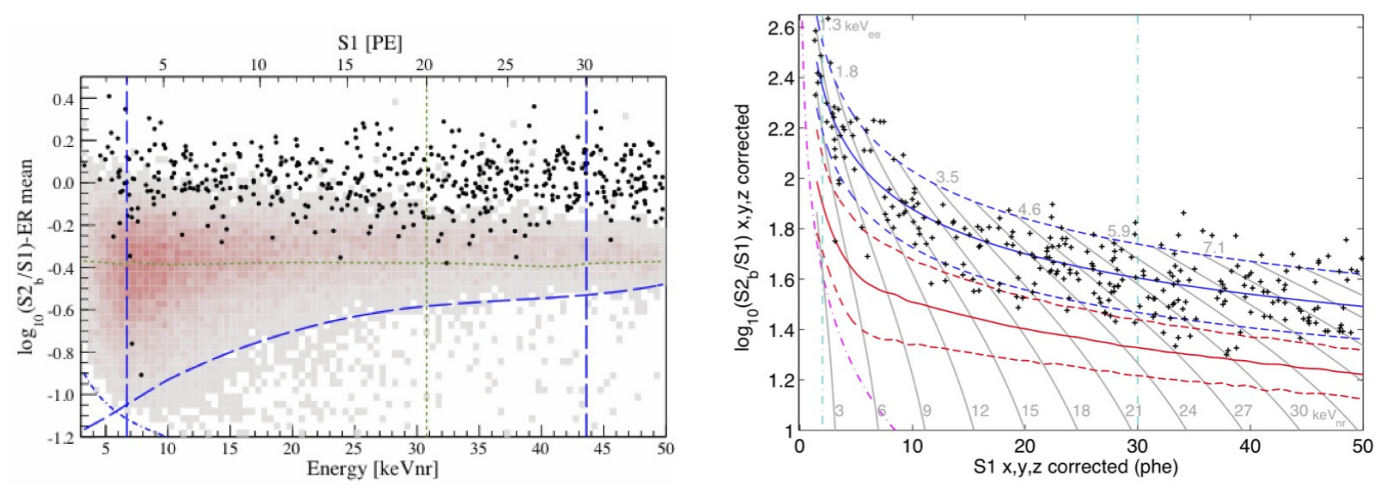

Figure 3. Ratio of secondary to primary signal (S2/S1) recorded in the WIMP-search data sample of XENON100 (left) and LUX (right) experiments, as a function of the S1 signal (see text). The vertical dashed lines represent the range used for the WIMP search. The red shaded distribution on the left represent the expected distribution for nuclear recoils, as measured in an neutron calibration. On the left, the red and blue regions correspond to the $80 \%$ efficient bands for nuclear and electronics recoils in LUX. The figures are taken from Ref. [14] and [13].

spectively. Only events occurring in the inner fiducial volume of $40 \mathrm{~kg}$ are considered (right pannel of Fig. 2).

Due to the high purity of the xenon, achieved by multiple purification cycles, and to the selfshielding capabilities of the detector shown in Fig. 2, the total events rate observed in the fiducial volume at low energy is $10^{-2}$ events per kg.day per keV in XENON100, and slightly above $10^{-3}$ in the larger, better self-shielded volume of LUX[13, 14]. Figure 3 shows the data in the selected fiducial volume of these experiments, corresponding to masses of xenon of $34 \mathrm{~kg}$ and $118 \mathrm{~kg}$ for XENON and LUX, respectively, and exposures of 225 and 85 days. The observations are compatible with the expected gamma-ray background. This technique provides the most stringent spin-independent limits for WIMP masses below $70 \mathrm{GeV} \cdot \mathrm{c}^{-2}$, as shown in Fig. 1. The XENON collaboration is building a larger detector with 1 ton of xenon for 2014 , with a sensitivity goal of $3 \times 10^{-11} \mathrm{pb}$. The LUX collaboration continues taking data and is preparing a multi-ton scale experiment, $\mathrm{LZ}$.

\section{Cryogenic crystal experiments}

\subsection{CDMS}

The experiment CDMS [10] in its most recent version (SuperCDMS@ Soudan) uses an array of fifteen $650 \mathrm{~g}$ cryogenic germanium detectors, with the simultaneous measurement of phonon and ionization signals. These detectors have two natural advantages: both phonon and ionization measurements have typical energy resolutions of one $\mathrm{keV}$ or less, and the radioactivity of hyperpure semiconductor crystals is extremely low. The ionization signal is collected using electrodes covering the surface of the crystal. The phonon signal is measured using eight arrays of $\sim 1000$ transition-edge tungsten sensors (TES), covering different zones on the two flat faces of the cylindrical detectors, operated at a temperatures between 54 and $62 \mathrm{mK}$. This system has a large acceptance to out-of-equilibrium phonons. As a result, the radial position of an interaction in the detector can be measured with a mm precision using the relative size of the phonon signals in each of the eight TES arrays, enabling the energy of the event to be reconstructed with a $\mathrm{keV}$ resolution. The TES arrays are interleaved with thin ionization 

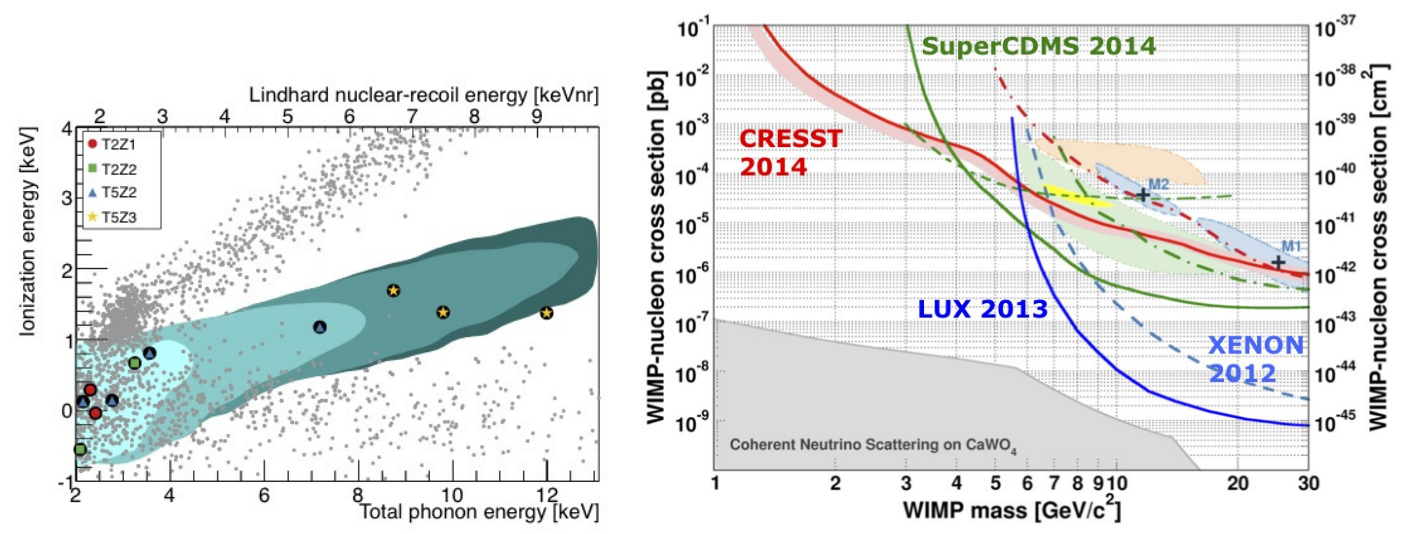

Figure 4. Left: Ionization signal versus recoil energy in the most recent CDMS data (see text: this figure is from Ref. [10]). The shaded areas correspond to the 95\% confidence regions expected for WIMP with masses of 5, 7,10 and $15 \mathrm{GeV} / \mathrm{c}^{2}$. The grey points are the events before the nuclear recoil selection, and the eleven colored points are those that pass the selection. Right: experimental limits on spin-independent WIMP-nucleon scattering cross-section as a function of WIMP mass, in the domain of low masses. This figure is adapted from Ref. [12]

electrodes biases at $\pm 2 \mathrm{~V}$ relative to the phonon sensors on each side of the detector. The charge readout can thus proceeds as in the EDELWEISS ID detectors (see section 4.2), as only bulk events will result in the collection of equal and opposite charge signals on the top and bottom electrodes. However, the charge collected on the TES cannot be readout in the present detector configuration. The time structure of the build-up of the athermal signal is also measured. The phonon signal rise time and its delay relative to the fast ionization signal are of the order of a few $\mu$ s. These time constants are systematically larger for phonons associated with nuclear recoils, relative to those arising from electron movements. The detectors thus provide three independent discrimination variables for the identification of nuclear recoil: the ionization yield relative to the total calorimetric energy measured by the phonon sensor, the charge collection balance between the two opposite side of the detector and the time structure of the phonon signal. CDMS has recently published results obtained with an exposure of $577 \mathrm{~kg}$.d, for a total fiducial mass of $6 \mathrm{~kg}$. Despite a much smaller target mass than the xenon-based experiments, the first physics run of these detectors yielded the most stringent limit on spin-independent interactions in the WIMP mass range from 4 to $6 \mathrm{GeV} / \mathrm{c}^{2}$ (Fig. 4). The collaboration is preparing for the next generation of arrays of more massive detectors (SuperCDMS at SNOLAB).

\subsection{EDELWEISS}

The experiment EDELWEISS [11] has set its most recent limits using an array of ten 400 g germanium cryogenic detectors, installed in the Laboratoire Souterrain de Modane (LSM). As CDMS, its detectors use the ratio of the ionization yield to the phonon signal to identify nuclear recoils. Here, the phonon measurement is provided by a simple GeNTD thermistance, glued to the detector. The signal is purely thermal, with a uniform response over the entire detector volume. The rejection of events with incomplete charge collection is based on the ionization measurement, using an interleaved electrode scheme described in Ref. [11]. The electrodes on the flat surfaces of the cylindrical detectors are replaced by concentric, annular interleaved electrodes, with a pitch of $2 \mathrm{~mm}$. With this "InterDigit" electrode design (ID), surface events are tagged by the presence of charge on two electrodes 
on the same side of the detector, resulting in a measured rejection factor for events due to the surface contamination by ${ }^{210} \mathrm{~Pb}$ of $\sim 10^{5}$. The EDELWEISS results have shown the high potential of the interleaved electrode scheme for the rejection of surface events, and has motivated a new detector design where the interleaved electrode pattern covers the entire surface of the detectors. This new design makes possible the increase of the total mass of the detectorto $800 \mathrm{~g}$ with no losses in rejection power. Tests of these new detectors are giving encouraging results, and an array of thirty-six $800 \mathrm{~g}$ detector is in operation since 2014. The fiducial target mass of more than $20 \mathrm{~kg}$ of germanium is the largest in operation. Further developments are being studied in the framework of the EURECA [20] collaboration, with a sensitivity goal of $10^{-11} \mathrm{pb}$. This project brings together the European efforts of the EDELWEISS and CRESST (see section 4.3) collaboration to built a ton-scale multi-target array of cryogenic detectors, in a growing collaboration with the project SuperCDMS.

\subsection{CRESST}

The CRESST collaboration [12] is also using cryogenic crystal detectors with phonon sensitivity, but in contrast with CDMS and EDELWEISS, it uses a scintillation signal instead of an ionization signal as a mean to identify nuclear recoils. The collaboration currently operates eighteen $300 \mathrm{~g} \mathrm{CaWO}_{4}$ detectors equipped with TES phonon sensors and operating at $10 \mathrm{mK}$. The scintillation photons are collected in a thin silicon wafer equipped with a TES sensor. Recently, the collaboration showed that one of its $250 \mathrm{~g}$ detector was able to achieve a $0.6 \mathrm{keV}$ threshold, with a background level so low that it resulted in the most stringent limits for spin-independent interactions for WIMP masses below $3 \mathrm{GeV} / \mathrm{c}^{2}$ (Fig. 4). This result is in large part due to the presence of three target nuclei in the same detector, the lightest one (oxygen) providing most of the sensitivity in that mass range. The CRESST collaboration is pursuing the development of these detectors, and is part of the EURECA efforts to plan the next generation of cryogenic detector experiment, in a growing collaboration with the project SuperCDMS.

\section{Searches beyond the Constrained SuperSymmetric Models}

While models inspired by the type of SUSY models that will be probed at LHC provide the main thrust for the current searches, some recent findings have prompted the appearance of alternate models that are sometime best probed by experiments with alternative designs. For instance, in models where spindependent processes dominate over much-suppressed spin-independent one, there is a strong interest for a detector with a target atom with a large nuclear spin, such as ${ }^{19} \mathrm{~F}$ [19]. There have been attempts to reconcile the observation of the annual rate modulation in DAMA with the stringent limits imposed by CDMS, XENON and many other experiments by invoking models where WIMPs have dominantly inelastic interactions [21]. This brought about alternative analyses of CDMS [10] and XENON [14] data. In another proposed model, the WIMP masses would be less than $10 \mathrm{GeV} \cdot \mathrm{c}^{-2}$ (lower than the LEP bounds for neutralinos), combined with the possibility - discarded by now [22] - that recoiling ions in NaI have a high probability for channeling. Another hint of a low-mass WIMP had been the report by the CoGeNT collaboration [23] of an excess rate below $2 \mathrm{keV}$ in a ionization-only detector (corresponding to $\sim 9 \mathrm{keV}$ if the reduced ionization yield for nuclear recoils is taken into account), a claim inconsistent with a majority of observations by other experiments.

\section{Conclusions}

Direct Dark Matter searches are crucial experiments to attest the presence of WIMPs in our environment, and provide a strategic complementarity with an eventual observation of SuperSymmetry 
at LHC. The experiment is apparently simple, but the required extremely low backgrounds are quite challenging and foster constant technological innovations. The need for observations with a variety of targets material motivates intense world-wide efforts in R\&D. The most sensitive technologies are, for now, double-phase xenon detectors (offering large masses, self-shielding and low thresholds) and cryogenic detectors (offering good performances in terms of resolution, low thresholds and discrimination). In the United States, these two technologies have been recently selected by DOE and NSF as part of the portfolio of "second generation" of direct dark matter experiments, with their collective sensitivity to both low and high mass WIMPs.

\section{References}

[1] G. Jungman, M. Kamionkowski, and K. Griest, Phys. Rep. 267, 195 (1996); G. Bertone, D. Hooper and J. Silk, Phys. Rep. 405, 279 (2005).

[2] J.D. Lewin and P.F. Smith, Astropart. Phys. 6, 87 (1996).

[3] M. Vogelsberger et al., MNRAS 395, 797 (2009); A. Green, Phys. Rev. D 66, 083003 (2002); C. Savage, K. Freese and P. Gondolo, Phys. Rev. D 74, 043531 (2006); P. Ullio and M. Kamionkowski, Journal of High Energy Physics, Issue 3, 49 (2001).

[4] R. Catena and P. Ullio, ArXiv:0907.0018.

[5] J. Billard et al., Phys. Rev. D 89, 023524 (2014).

[6] J.L. Feng et al., Planning the Future of U.S. Particle Physics (Snowmass 2013): Chapter 4: Cosmic Frontier, Arxiv:1401.6085 (2014).

[7] S. Ahlen et al., International Journal of Modern Physics A 25, 1 (2010).

[8] R. Bernabei et al., Eur. Phys. J. C 67, 39 (2010).

[9] A. Benoit et al., Nucl. Instr. Meth. Phys. Res. A 577, 558 (2007) and references therein.

[10] R. Agnese et al., Phys. Rev. Lett. 112, 241302 (2014); Z. Ahmed et al., Science 327, 1619 (2010).

[11] E. Armengaud et al., Phys. Lett. B 702, 329 (2011); A. Broniatowski et al., PLB 681, 305 (2009).

[12] G. Angloher et al., Astroparticle Physics 31, 270 (2009).

[13] D.S. Akerib et al., Phys. Rev. Lett. 112, 091303 (2014).

[14] E. Aprile et al., Phys. Rev. Lett. 109, 181301 (2012).

[15] D.Y. Akimov et al., Phys. Lett. B 709, 14 (2012).

[16] M.G. Boulay and A. Hime, Astropart. Physics 25, 179 (2006); P. Benetti et al., Astropart. Phys. 28, 495 (2008).

[17] H.S. Lee et al., Phys. Rev. Lett. 99 (2007) 091301.

[18] A. A. Aguilar-Arevalo et al. (The DAMIC Collaboration), Proceeding of the ICRC2013 conference, arXiv:1310.6688 (2013).

[19] E. Behnke et al., Phys. Rev. D 86, 052001 (2012); S. Archambault et al., Phys. Lett. B 711, 153 (2012); M. Felizardo et al., Phys. Rev. Lett. 108, 201302 (2012); C.E. Dahl et al., Phys. Rev. Lett. 108, 259001 (2012).

[20] H. Kraus et al., J. Phys. Conf. Ser. 39 (2006) 139.

[21] S. Chang, G.D. Kribs, D. Tucker-Smith and N. Weiner, Phys. Rev. D 79 (2009) 043513; D. Smith and N. Weiner, Phys. Rev. D 64 (2001) 043502.

[22] N. Bozorgnia, G. B. Gelmini and P. Gondolo, JCAP 11 (2010) 019.

[23] C.E. Aalseth et al., Phys. Rev. D 88, 012002 (2013). 